\title{
Coating techniques of metal chambers for remote catalytic chemical vapor deposition applications
}

\author{
Hironobu Umemoto ${ }^{\text {a) }}$ \\ Faculty of Engineering, Shizuoka University, Johoku, Naka, Hamamatsu, Shizuoka \\ 432-8561, Japan
}

Shota Setoguchi, Hitoshi Uemura, and Hideki Matsumura

School of Materials Science, Japan Advanced Institute of Science and Technology, Asahidai, Nomi, Ishikawa 923-1292, Japan

To obtain information for remote catalytic chemical vapor deposition, we constructed a two-chamber system in which the decomposition and deposition areas were separated. $\mathrm{H}_{2}$ molecules were decomposed on a heated tungsten filament in the first area and the $\mathrm{H}$ atoms produced were carried to the second area, where the absolute H-atom densities were measured by a vacuum-ultraviolet laser absorption technique. The gas pressure in the decomposition area was maintained 10 times higher than that in the deposition area by mounting a diffusion barrier to prevent the possible back-diffusion of the material gases. The inner walls of the decomposition area as well as the diffusion barrier were coated with $\mathrm{SiO}_{2}$ or polytetrafluoroethene to minimize the recombination of $\mathrm{H}$ atoms. The $\mathrm{H}$-atom density could be increased by one order of magnitude by such coatings, and it is shown that $\mathrm{H}$-atom densities in the order of $10^{12} \mathrm{~cm}^{-3}$ can be achieved in the deposition area in the present system.

\footnotetext{
a) Electronic mail: thumemo(at)ipc.shizuoka.ac.jp

Materials: $\mathrm{H}_{2}, \mathrm{SiO}_{2}$, polytetrafluoroethene
} 


\section{INTRODUCTION}

In catalytic chemical vapor deposition (Cat-CVD in short, often called hot-wire CVD or hot-filament CVD) processes, the material gases are decomposed to radical species on resistively heated metal surfaces. ${ }^{1,2}$ One of the problems in Cat-CVD is the poisoning of the catalyzer surfaces, when more than two kinds of material gases are used. For example, it is easy to decompose $\mathrm{NH}_{3}$ catalytically more than $50 \%$ in a pure $\mathrm{NH}_{3}$ system. However, when a tiny amount of $\mathrm{SiH}_{4}$ is introduced, the decomposition efficiency of $\mathrm{NH}_{3}$ decreases by one order of magnitude. ${ }^{3-5}$

One idea for overcoming this poisoning problem is remote Cat-CVD, in which decomposition and deposition areas are separated. ${ }^{6}$ In the decomposition area, for example, pure $\mathrm{NH}_{3}$ is decomposed to $\mathrm{NH}_{2}$ and $\mathrm{H}$ and these radical species are transported to the deposition area, where $\mathrm{SiH}_{4}$ is introduced. $\mathrm{SiH}_{3}$ radicals can be produced in the reactions of $\mathrm{H}$ and $\mathrm{SiH}_{4}$ and contribute to the deposition of silicon nitride $\left(\mathrm{SiN}_{\mathrm{x}}\right)$ films. ${ }^{7,8}$ In remote Cat-CVD, one also expects to prepare hydrogenated amorphous silicon (a-Si:H) films with fewer dangling bonds. In conventional Cat-CVD, the main decomposition product of $\mathrm{SiH}_{4}$ is $\mathrm{Si}^{9-14} \mathrm{Si}$ atoms thus produced will react with $\mathrm{SiH}_{4}$ to produce $\mathrm{Si}_{2} \mathrm{H}_{4} \cdot{ }^{15,16}$ Such heavy species are regarded as the source of dangling bonds. If $\mathrm{SiH}_{3}$ radicals can be produced selectively by a reaction between $\mathrm{H}$ and $\mathrm{SiH}_{4}$ without co-producing other active species in remote Cat-CVD, that should be beneficial in fabricating a-Si:H with fewer dangling-bond defects. ${ }^{17,18}$ To put this remote Cat-CVD technique into practice, it is necessary to minimize the recombination probabilities of radical species during the transportation. Recently, we have shown that $\mathrm{SiO}_{2}$ coating, as well as polytetrafluoroethene coating, is useful to reduce the recombination probabilities of $\mathrm{H}$ atoms on metal chamber walls by using a single-chamber system. ${ }^{19}$

In the present work, to verify the validity of such coating techniques, we have 
constructed a two-chamber system in which the decomposition and deposition areas were separated. $\mathrm{H}_{2}$ molecules were decomposed in the first area and the $\mathrm{H}$ atoms produced were carried to the second area, where the absolute H-atom densities were measured. A diffusion barrier, an Al plate with many pinholes, was installed between the decomposition and deposition areas. Without such a barrier, the pressure gradient will be too small to prevent the back-diffusion of the material gases.

\section{EXPERIMENT}

The experimental apparatus and the procedure were similar to those described elsewhere except that the chamber system was modified for remote Cat-CVD. ${ }^{4,21}$ Figure 1 illustrates schematically the present chamber system. The main chamber (deposition chamber) was the same as that used in our previous studies. The internal diameter was $45 \mathrm{~cm}$ and it was evacuated with a turbo molecular pump (Osaka Vacuum, TG1000). The side chamber (decomposition chamber) was $18 \mathrm{~cm}$ in internal diameter and $40 \mathrm{~cm}$ in length. A diffusion barrier was installed between these two chambers. The diffusion barrier was made of $\mathrm{Al}$ and was $16 \mathrm{~cm}$ in diameter and $1.0 \mathrm{~mm}$ in thickness. 553 holes of $0.1-\mathrm{mm}$ diameter were drilled in this disk. The distance between the catalyzer and the diffusion barrier was $22 \mathrm{~cm}$, while that between the barrier and the detection zone was $28 \mathrm{~cm}$. The catalyzer was a tungsten wire (30 $\mathrm{cm}$ in length and $0.38 \mathrm{~mm}$ in diameter) and was resistively heated using a DC power supply (Takasago, EX-1125H2). $\mathrm{H}_{2}$ gas was introduced into the side chamber and decomposed to $\mathrm{H}$ atoms on the heated tungsten filament. The absolute densities of $\mathrm{H}$ atoms in the main chamber were evaluated by a vacuum-ultraviolet laser absorption technique. The output of a dye laser (Lumonics, HD-500) pumped with a Nd:YAG (YAG denotes yttrium aluminum garnet) laser (Quanta-Ray, PRO-190) at $729.6 \mathrm{~nm}$ was doubled in frequency by a $\beta-\mathrm{BaB}_{2} \mathrm{O}_{4}$ crystal and then tripled by a mixture of $\mathrm{Kr}$ 
and Ar to produce Lyman- $\alpha$ light at $121.6 \mathrm{~nm}$. The typical pulse energy of the laser at the doubled stage $(364.8 \mathrm{~nm})$ was $10 \mathrm{~mJ}$. The 364.8-nm light was focused into the tripling cell by a 150-mm focal-length lens and the resultant 121.6-nm light was collimated with an $\mathrm{MgF}_{2}$ lens. After passing through the chamber, the laser beam entered a detection vessel filled with $130 \mathrm{~Pa}$ of $\mathrm{NO}$. The $\mathrm{NO}^{+}$ion current was measured with a boxcar averager-gated integrator system (Stanford Research Systems, SR250/SR280). The $\mathrm{H}_{2}$ pressure at the main chamber was kept constant at 5.6 Pa. The flow rate of $\mathrm{H}_{2}$ was changed between 100 and $300 \mathrm{sccm}\left(1 \mathrm{sccm}=6.9 \times 10^{-7} \mathrm{~mol}\right.$ $\mathrm{s}^{-1}$ ) by using a mass flow controller (STEC, SEC-400MK3). The $\mathrm{H}_{2}$ pressure at the side chamber was $55 \mathrm{~Pa}$ when the flow rate was $150 \mathrm{sccm}$.

The inner walls of the decomposition chamber as well as the diffusion barrier were coated with either $\mathrm{SiO}_{2}$ or polytetrafluoroethene. The procedure of $\mathrm{SiO}_{2}$ coating was similar to that described previously. ${ }^{19}$ After smearing a xylene solution of low-temperature hardening-type perhydropolysilazane, the chamber was heated at 420 $\mathrm{K}$ in a furnace for 12 hours. The film thickness was less than $1 \mu \mathrm{m}$. The polytetrafluoroethene coating was carried out by Cat-CVD of hexafluoropropeneoxide (SynQuest Laboratories). The procedure was similar to that developed by Gleason and coworkers. ${ }^{22}$ The flow rate of hexafluoropropeneoxide was $50 \mathrm{sccm}$, while the pressure was $16 \mathrm{~Pa}$. A nichrome wire $80 \mathrm{~cm}$ in length and $0.5 \mathrm{~mm}$ in diameter was used as a catalyzer. The catalyzer temperature was $1050 \mathrm{~K}$ and the deposition time was 5 hours. The estimated film thickness is $5 \mu \mathrm{m} .^{23}$

$\mathrm{H}_{2}$ (Takachiho, 99.99995\%), Kr (Nihon Sanso, 99.995\%), Ar (Nihon Sanso, 99.9995\%), and NO (Nihon Sanso, 99\%) were used from cylinders without further purification.

\section{RESULTS}


Figure 2 shows the relationships between the $\mathrm{H}$-atom density in the deposition chamber and the reciprocal of the catalyzer temperature. The measurements were carried out under coated and uncoated conditions, with and without a diffusion barrier. The $\mathrm{H}_{2}$ flow rate and the pressure in the deposition chamber were kept at $150 \mathrm{sccm}$ and 5.6 $\mathrm{Pa}$, respectively. The $\mathrm{H}_{2}$ pressure in the decomposition chamber was $55 \mathrm{~Pa}$ when a diffusion barrier was installed. It is not clear if such a large pressure gradient is necessary to prevent the back-diffusion from the deposition chamber when a second material gas is introduced, but this pressure ratio should be large enough. The $\mathrm{H}_{2}$ flow rate dependence of the $\mathrm{H}$-atom density was examined, but the dependence was minor when the pressure in the deposition chamber was fixed.

Under coated conditions, especially when coated with polytetrafluoroethene, the H-atom density is much higher than that under uncoated conditions. By extrapolating the plots in Fig. 2, it is possible to estimate the $\mathrm{H}$-atom densities at higher catalyzer temperatures. In absorption measurements, the dynamic range is not wide and the catalyzer temperature could not be changed widely when other parameters were fixed. In the absence of a diffusion barrier, the $\mathrm{H}$ atom density is estimated to be as high as $1.0 \times 10^{14} \mathrm{~cm}^{-3}$ when the catalyzer temperature is $2600 \mathrm{~K}$. Actually, by using a two-photon laser-induced fluorescence technique, we have confirmed the production of such high-density $\mathrm{H}$ atoms. ${ }^{20}$ In the presence of a diffusion barrier, the $\mathrm{H}$-atom densities at $2600 \mathrm{~K}$ are estimated to be $2.0 \times 10^{11}$ and $5.6 \times 10^{11} \mathrm{~cm}^{-3}$ for $\mathrm{SiO}_{2}$ and polytetrafluoroethene coated conditions, respectively. In other words, it is expected that the H-atom density can be increased to the order of $10^{12} \mathrm{~cm}^{-3}$ even in the presence of a diffusion barrier. It may be possible to increase the H-atom density by a factor of two or three by using longer catalyzers, if a water-cooled chamber is used to prevent the temperature rise on chamber walls. It may also be possible to increase the catalyzer temperature over $2600 \mathrm{~K}$ since metal contamination must be less critical in 
remote Cat-CVD.

In the present study, coating with polytetrafluoroethene was found to be better for increasing the $\mathrm{H}$-atom density than coating with $\mathrm{SiO}_{2}$, although no superiority was found in our previous study. ${ }^{19}$ This difference may be ascribed to the difference in the surface smoothness of the polytetrafluoroethene films. The polytetrafluoroethene film prepared by Cat-CVD in the present study is smoother, although less adhesive to stainless-steel walls, than that employed in our previous study.

\section{DISCUSSION}

The slopes of the linear plots shown in Fig. 2 are different for those with and without a diffusion barrier. The slope without a barrier is larger and agrees with those reported previously by ourselves, as well as by others. ${ }^{20,24}$ The difference between the slopes in the presence and absence of a barrier may be ascribed to that in the $\mathrm{H}$-atom recombination rates on chamber walls. In the presence of a diffusion barrier, the $\mathrm{H}$-atom density in the decomposition chamber must be high, and the removal rate of $\mathrm{H}$ atoms should be controlled by the recombination rate on chamber walls, which is expected to increase with the square of the H-atom density. In the absence of a barrier, the diffusional loss of $\mathrm{H}$ atoms, the rate of which must be directly proportional to the H-atom density, must be more dominant. Then, in the presence of a diffusion barrier, the removal rate of $\mathrm{H}$ atoms must increase more rapidly with the increase in the production rate of $\mathrm{H}$ atoms than that in the absence of a barrier. In addition, the rise in the wall temperature with the increase in the catalyzer temperature must be larger when a diffusion barrier is installed. The H-atom recombination process is, of course, exothermic and brings about the rise in the surface temperature. Because the recombination probability of $\mathrm{H}$ atoms increases with the surface temperature, ${ }^{25-27}$ the recombination rate may increase spirally. 
This vicious spiral in the recombination rate and the surface temperature may be avoided by using a larger decomposition chamber with a cooling system. The heat radiation must be better in a larger chamber. In addition, when a large decomposition chamber is used, only radical species near the central part of the chamber can be sampled. Of course, the radical density at the central part must be higher than that near the walls. If the $\mathrm{H}$-atom density can be increased by one order by employing such modifications, it may be possible to use remote Cat-CVD techniques in practical processes.

\section{CONCLUSIONS}

The future prospect of a remote catalytic chemical vapor deposition technique, in which the decomposition area of material gases and the deposition area of radical species were separated, was discussed on the basis of the absolute H-atom density measurements. It is shown that coating of the inner walls of the chamber with $\mathrm{SiO}_{2}$ or polytetrafluoroethene is useful to reduce the surface recombination loss of radical species and to maintain high radical densities. It is possible to increase the H-atom density in the deposition chamber up to the order of $10^{12} \mathrm{~cm}^{-3}$. By using a water-cooled decomposition chamber whose diameter is comparable to that of the deposition chamber, it should be possible to further increase the radical density. By employing such a system, remote Cat-CVD can be a promising technique for preparing thin films.

\section{ACKNOWLEDGMENT}

This work was partially funded by the Grant-in-Aid for Science Research (Nos. 17550009 and 19550015) from the Japan Society for the Promotion of Science. 


\section{References}

${ }^{1}$ H. Matsumura, Jpn. J. Appl. Phys. 37, 3175 (1998).

${ }^{2}$ H. Matsumura, A. Masuda, and H. Umemoto, Thin Solid Films 501, 58 (2006).

${ }^{3}$ H. Umemoto, T. Morimoto, M. Yamawaki, Y. Masuda, A. Masuda, and H. Matsumura, Thin Solid Films 430, 24 (2003).

${ }^{4}$ H. Umemoto, K. Ohara, D. Morita, T. Morimoto, M. Yamawaki, A. Masuda, and H. Matsumura, Jpn. J. Appl. Phys. 42, 5315 (2003).

${ }^{5}$ S.G. Ansari, H. Umemoto, T. Morimoto, K. Yoneyama, A. Izumi, A. Masuda, and H. Matsumura, Thin Solid Films 501, 31 (2006).

${ }^{6}$ H. Umemoto and H. Matsumura, Thin Solid Films 516, 500 (2008).

${ }^{7}$ N.L. Arthur and L.A. Miles, J. Chem. Soc. Faraday Trans. 93, 4259 (1997).

${ }^{8}$ N.L. Arthur, I.A. Cooper, A. Czerwinski, and L.A. Miles, Thin Solid Films 368, 176 (2000).

${ }^{9}$ Y. Nozaki, K. Kongo, T. Miyazaki, M. Kitazoe, K. Horii, H. Umemoto, A. Masuda, and H. Matsumura, J. Appl. Phys. 88, 5437 (2000).

${ }^{10}$ S. Tange, K. Inoue, K. Tonokura, and M. Koshi, Thin Solid Films 395, 42 (2001).

${ }^{11}$ K. Tonokura, K. Inoue, and M. Koshi, J. Non-Cryst. Solids 299-302, 25 (2002).

${ }^{12}$ H.L. Duan, G.A. Zaharias, and S.F. Bent, Thin Solid Films 395, 36 (2001).

${ }^{13}$ H.L. Duan, G.A. Zaharias, and S.F. Bent, Appl. Phys. Lett. 78, 1784 (2001).

${ }^{14}$ J.K. Holt, M. Swiatek, D.G. Goodwin, and H.A. Atwater, J. Appl. Phys. 92, 4803 (2002).

${ }^{15}$ S. Nakamura and M. Koshi, Thin Solid Films 501, 26 (2006).

${ }^{16}$ S. Nakamura, A. Matsugi, A. Susa, and M. Koshi, Thin Solid Films 516, 517 (2008).

${ }^{17}$ A. Matsuda, J. Non-Cryst. Solids 338-340, 1 (2004).

${ }^{18}$ A. Matsuda, Jpn. J. Appl. Phys. 43, 7909 (2004).

${ }^{19}$ S.G. Ansari, H. Umemoto, T. Morimoto, K. Yoneyama, A. Masuda, H. Matsumura, 
M. Ikemoto, and K. Ishibashi, J. Vac. Sci. Technol. A 23, 1728 (2005).

${ }^{20}$ H. Umemoto, K. Ohara, D. Morita, Y. Nozaki, A. Masuda, and H. Matsumura, J. Appl. Phys. 91, 1650 (2002).

${ }^{21}$ T. Morimoto, H. Umemoto, K. Yoneyama, A. Masuda, H. Matsumura, K. Ishibashi, H. Tawarayama, and H. Kawazoe, Jpn. J. Appl. Phys. 44, 732 (2005).

${ }^{22}$ K.K.S. Lau, J.A. Caulfield, and K.K. Gleason, Chem. Mater. 12, 3032 (2000).

${ }^{23}$ H. Yasuoka, M. Yoshida, K. Sugita, K. Ohdaira, H. Murata, and H. Matsumura, Thin Solid Films 516, 687 (2008).

${ }^{24}$ W. Zheng and A. Gallagher, Surf. Sci. 600, 2207 (2006).

${ }^{25}$ A. Rousseau, A. Granier, G. Gousset, and P. Laprince, J. Phys. D 27, 1412 (1994).

${ }^{26}$ B.J. Wood and H. Wise, J. Phys. Chem. 66, 1049 (1962).

${ }^{27}$ Y.C. Kim and M. Boudart, Langmuir 7, 2999 (1991). 


\section{Figure Captions}

FIG. 1. Schematic diagram of the present apparatus for remote Cat-CVD.

FIG. 2. Logarithmic plots of the H-atom density as a function of the reciprocal of catalyzer temperature, $T_{\text {cat }}$. The $\mathrm{H}_{2}$ flow rate was $150 \mathrm{sccm}$. The pressure in the decomposition chamber was $55 \mathrm{~Pa}$ when a diffusion barrier was installed, while that in the deposition chamber was 5.6 Pa. The wall coating materials were $\mathrm{SiO}_{2}(\bullet)$ and polytetrafluoroethene $(\square)$, while $\Delta$ represents the results without coatings, that is, bare stainless-steel walls. The results obtained without a diffusion barrier are represented by 


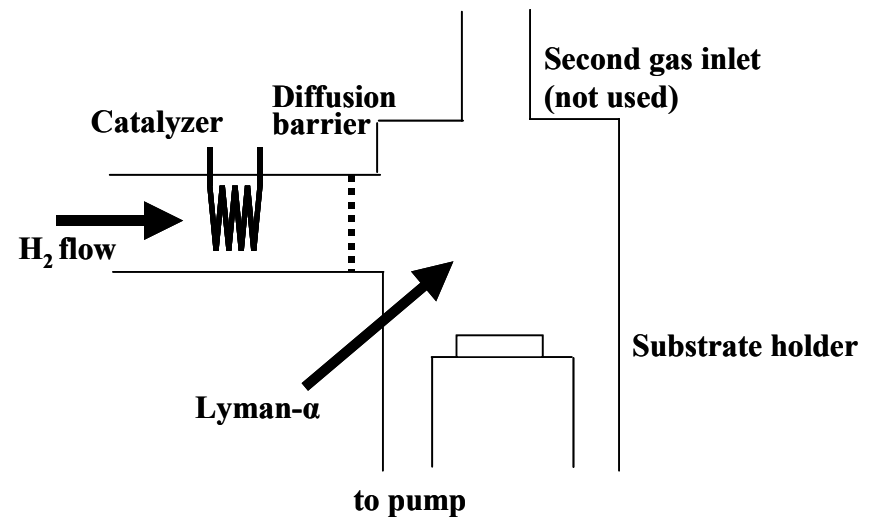

Fig. $1 \mathrm{H}$. Umemoto et al. 


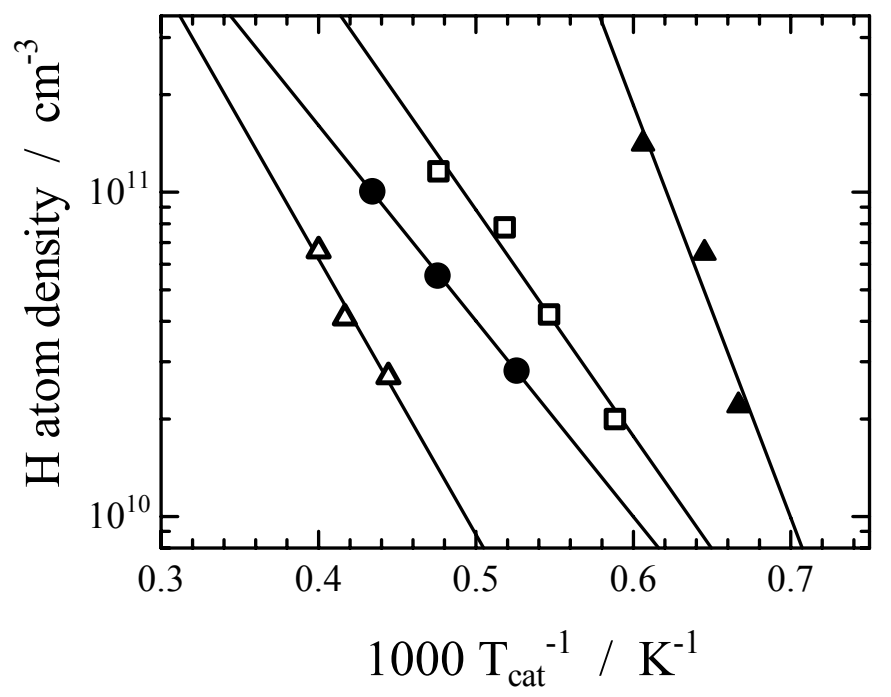

Fig. 2. H. Umemoto et al. 